Research Paper

\title{
RHOV promotes lung adenocarcinoma cell growth and metastasis through JNK/c-Jun pathway
}

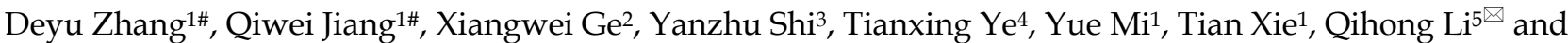 \\ Qinong $\mathrm{Ye}^{1}$
}

1. Department of Medical Molecular Biology, Beijing Institute of Biotechnology, Beijing 100850, P.R. China.

2. Department of Oncology, Chinese PLA General Hospital, Beijing 100853, P.R. China.

3. Medical College, Guizhou University, Guiyang 550025, P.R. China.

4. College of Medicine, Yanbian University, Yanji 133000, P.R. China.

5. Department of Stomatology, The Fifth Medical Centre, Chinese PLA General Hospital, Beijing 100071, P.R. China.

\#These authors contributed equally to this work.

$\triangle$ Corresponding authors: Qinong Ye, Department of Medical Molecular Biology, Beijing Institute of Biotechnology, Beijing 100850, China. Phone: 8610-66932183; Fax: 8610-88271216; E-mail: yeqn66@yahoo.com; Qihong Li, E-mail: liqihong@126.com.

(C) The author(s). This is an open access article distributed under the terms of the Creative Commons Attribution License (https://creativecommons.org/licenses/by/4.0/). See http://ivyspring.com/terms for full terms and conditions.

Received: 2021.03.01; Accepted: 2021.06.08; Published: 2021.06.22

\begin{abstract}
Lung adenocarcinoma (LUAD) is a common type of lung cancer with high frequent metastasis and a high death rate. However, genes responsible for LUAD metastasis are still largely unknown. Here, we identify an important role of ras homolog family member $\mathrm{V}$ (RHOV) in LUAD metastasis using a combination of bioinformatic analysis and functional experiments. Bioinformatic analysis shows five hub LUAD metastasis driver genes (RHOV, ZIC5, CYP4B1, GPR 18 and TCP10L2), among which RHOV is the most significant gene associated with LUAD metastasis. High RHOV expression predicted shorter overall survival in LUAD patients. RHOV overexpression promotes proliferation, migration, and invasion of LUAD cells, whereas RHOV knockdown inhibits these biological behaviors. Moreover, knockdown of RHOV suppresses LUAD tumor growth and metastasis in nude mice. Mechanistically, RHOV activates Jun N-terminal Kinase (JNK)/c-Jun signalling pathway, an important pathway in lung cancer development and progression, and regulates the expression of markers of epithelial-to-mesenchymal transition, a process involved in cancer cell migration, invasion and metastasis. RHOV-induced malignant biological behaviors are inhibited by pyrazolanthrone, a JNK inhibitor. Our findings indicate a critical role of RHOV in LUAD metastasis and may provide a biomarker for prognostic prediction and a target for LUAD therapy.
\end{abstract}

Key words: Lung adenocarcinoma, metastasis, JNK/c-Jun pathway, RHOV, bioinformatics

\section{Introduction}

Lung carcinoma is one of malignant cancers with the highest incidence and the worst prognosis in worldwide [1]. Most lung carcinoma patients are diagnosed at advanced stage with metastatic disease $[2,3]$. Among all lung carcinoma subtypes, lung adenocarcinoma (LUAD) is the most common and aggressive subgroup [2]. The pathophysiological mechanisms of high metastasis rate of LUAD remain poorly understood. Several factors, such as immune escape [4], genetic mutation [5], and pathological and molecular alterations [6], were reported to be associated with metastasis of LUAD. Considering the inadequate understanding of LUAD metastasis, we need to explore novel targets.

Ras Homolog (Rho) GTPases belong to the Ras superfamily of small GTPases, and play important roles in regulation of cell cycle, cell adhesion and migration [7]. Ras homolog family member $\mathrm{V}$ (RHOV) is considered an atypical member of Rho GTPases because of unique $\mathrm{N}$-terminal and C-terminal regions. RHOV was reported to promote neural crest development during vertebrate embryogenesis [8]. In zebrafish, RHOV has ability to activate (p21-activated kinases) PAK1, thus promoting early vertebrate development [9]. Although RHOV expression was shown to be upregulated in lung carcinoma [10], the biological function of RHOV in cancer is unclear.

In this study, we demonstrate that RHOV may be associated with metastasis in LUAD through bioinformatics. We show that silencing of RHOV 
reduces LUAD cell proliferation, migration, invasion, and metastasis in vitro and in vivo. Mechanistically, RHOV activates Jun N-terminal Kinase (JNK)/c-Jun signalling pathway, a critical pathway in lung cancer development and progression, and modulates the expression of markers of epithelial-to-mesenchymal transition (EMT), a process involved in cancer cell migration, invasion and metastasis.

\section{Materials and Methods}

\section{Plasmids, cell lines and reagents}

The FLAG-tagged RHOV eukaryotic expression vector was constructed using the PCR-amplified RHOV fragment and pcDNA3.0 (Invitrogen) with FLAG tag. The lung cancer cell lines A549 and H1299 were purchased from the American Type Culture Collection. The target sequence of RHOV shRNA was 5'-GGCTATTCTCAGTGCCATT-3'. Lentivirus was produced using pSIH-H1-Puro (System Biosciences) carrying RHOV shRNA according to the manufacturer's instructions, and was infected into A549 and H1299 lung cancer cells to generate individual clones. Anti-JNK, anti-phospho-JNK (pJNK), anti-c-Jun, anti-phospho-c-Jun and anti-RHOV were purchased from Proteintech; Anti-E-cadherin, anti-N-cadherin, anti-Snail and anti-Slug were purchased from BD Biosciences.

\section{Data collection}

The data of 535 LUAD tissue samples and 59 adjacent tissue samples were obtained from The Cancer Genome Atlas (TCGA-LUAD, www. cancergenome.nih.gov) [11]. The AJCC tumor-nodemetastasis (TNM) staging system was utilized in definition of metastatic predisposition. Two hundred thirty one LUAD tissues with $\mathrm{N} 0$ and M0 stage were identified as non-metastatic predisposition, and 185 LUAD tissues with N1-3 or M1 stage were identified as metastatic predisposition.

\section{Identification of metastasis driver genes (MDGs) and construction of Protein-protein interaction (PPI) network}

To investigate differentially expressed genes (DEGs) between LUAD tissues with non-metastatic predisposition and LUAD tissues with metastatic predisposition in TCGA-LUAD dataset, we used the limma R package [12]. DEGs were set as follows: False Discovery Rate (FDR) $<0.05$ and $\mid \log _{2}$ fold change (FC) $\mid>1$. Among DEGs, a PPI network of MDGs was built by the Search Tool for the Retrieval of Interacting Genes (STRING, www.string-db.org/), and was organized and visualized by Cytoscape software.

\section{Construction and evaluation of LUAD-specific prognostic model}

Univariable Cox regression analysis was performed to explore the relationships between MDGs and overall survival (OS) of 505 LUAD samples from TCGA-LUAD dataset. Multivariate Cox regression analysis was performed to establish a prognostic signature for each LUAD sample. The risk score was calculated as follows: Risk score = $\sum \beta i \times \operatorname{expRNAi}[13]$. The cases were ranked into lowand high-risk groups based on the median score, and the survival curves were plotted. Receiver operating characteristic (ROC) curve analysis was utilized to assess the prognostic signature.

\section{Analysis of Prognostic Value}

Gene Expression Profiling Interactive Analysis (GEPIA, http://gepia.cancer-pku.cn/) was used for analysis of the associations between MDGs and overall survival (OS) or disease-free survival (DFS) of LUAD patients from TCGA-LUAD dataset.

\section{Cell culture and Transfection}

A549 and H1299 cells were routinely cultured in Ham's F12 Medium or RPMI-1640 Medium containing $10 \%$ fetal bovine serum (FBS) at $37^{\circ} \mathrm{C}$ in a humidified atmosphere with 5\% CO2. For plasmid transfection, cells were inoculated into 6-well plates at a cell density of about $70-80 \%$ and transfected after adherence to the wall. The reagents for transfection of plasmids were Lipofectamine 3000 (Promega) and were used according to the manufacturer's instructions. Cell culture medium was replaced after 4-6 h, and the transfected cells were collected after 24-48 h.

\section{Cell proliferation and colony formation assays}

Cell proliferation assays were measured using the CCK-8 Kit (Dojindo Laboratories, Japan) according to the manufacturer's instructions. Cells were seeded in 96-well plates at a density of $3 \times 10^{3} /$ well, and 3 parallel wells were set in each group. DMEM containing 10\% CCK8 reagent was added to each well at the same time point every day, and cultured at $37^{\circ} \mathrm{C}$ for $1 \mathrm{~h}$. The OD450nm values were measured using a microplate reader. For colony formation assays, cells were inoculated in 6-well plate with $3 \times 10^{3}$ cells. After about two weeks, the colonies were treated with $4 \%$ paraformaldehyde and $0.5 \%$ crystal stained purple was kept for 30 minutes. Colonies larger than $1.5 \mathrm{~mm}$ in diameter were counted.

\section{Wound-healing and transwell assays}

Cells were inoculated in 6-well plates for cell 
transfection. After the cell density reached $80-90 \%$, the pipette head was used for scratching. The floating cells were washed with PBS buffer. Cultured cells were grown for $16 \mathrm{~h}$ to allow wound closure. The wound healing rates were measured and compared to the width at $0 \mathrm{~h}$. Transwell assay was used to detect cell invasion ability. Cells were collected and suspended in serum-free medium. Six hundred microliters of medium containing 20\% FBS was added to the 24-well plate, and about $200 \mathrm{uL}$ cell suspension was added to the upper chamber with matrigel, and cultured for $16 \mathrm{~h}$ in an incubator at $37^{\circ} \mathrm{C}$. The chamber was fixed in $4 \%$ paraformaldehyde and then stained in $0.1 \%$ crystal violet solution for 30 minutes, respectively. A cotton swab was used to wipe the cells and matrigel out of the chamber. The images were observed under a microscope, and the number of invasive cells was calculated using the Image J software.

\section{Tumor growth and metastasis in nude mice}

Animal experiments were performed with protocols approved by the Institutional Animal Care Committee at Beijing Institute of Biotechnology. Ten million A549 cells stably expressing RHOV shRNA or control shRNA were injected subcutaneously in the right flank of each nude mice. Tumor size was examined at the indicated times. The mice were euthanized at the indicated time. Excised tumors were perserved in liquid nitrogen.

For tumor metastasis analysis, $1 \times 10^{7} \mathrm{~A} 549$ cells stably expressing RHOV shRNA or control shRNA were injected into the tail vein of each nude mice. After euthanasia at the indicated time, all lung metastatic foci were investigated histologically.

\section{Statistical analysis}

SPSS 19.0 and GraphPad Prism 8.0 were used for statistical analysis. Student's $t$ test was applied to data comparison between two groups. Analysis of variance (ANOVA) was used for comparison among several groups. Student's $t$ test, Chi-square test and MannWhitney Test was adopted to analyze the relationship between clinicopathological characteristics and metastatic predisposition in LUAD patients. All experiments were independently repeated for 3 times, and the data were expressed as mean \pm SD. $P<0.05$ was the threshold of statistical significance.

\section{Results}

\section{Identification of RHOV as a critical gene associated with LUAD metastasis}

Two hundred thirty one LUAD tissues with non-metastatic predisposition and 185 LUAD tissues with metastatic predisposition from the TCGA-LUAD dataset were analyzed. The main clinical characteristics were presented in Table S1. Compared to non-metastasis group, 30 genes were upregulated and 665 genes were downregulated in metastasis group (Figure 1A). In order to screen out the most important and largest functional module, these differentially expressed genes (DEGs) were further selected as metastasis driver genes (MDGs) by the STRING online database, and a PPI network for 83 MDGs was built (Figure 1B).

These 83 MDGs were chosen for further analysis of the association between MDGs and patients' outcomes. Univariable Cox regression analysis was utilized to identified 19 MDGs with significant prognostic values (Figure 1C). Subsequently, multivariate Cox regression analysis was utilized to screen out five hub MDGs: RHOV, Zic family member 5 (ZIC5), cytochrome P450 family 4 subfamily B member 1 (CYP4B1), G protein-coupled receptor 18 (GPR18), and t-complex 10 like 2 (TCP10L2). A five-mRNA based prognostic signature was constructed and the prognostic risk score for each LUAD patient was as follows: $\left(0.137^{*} \mathrm{RHOV}+0.356^{*}\right.$ ZIC5 - 0.083* CYP4B1 - 0.29* GPR18 - 4.37* TCP10L2). The concordance index of this formula was 0.68, indicating a certain predictive effect. Among these five MDGs, RHOV was the most significant independent prognostic factor $(P$ value $=0.003$, Hazard ratio $=1.147$ ), and may play an essential role in metastasis and prognosis of LUAD patients (Figure 1D).

Moreover, we divided 504 LUAD patients into low- and high-risk groups based on the median risk score. Survival analysis and the expression of five MDGs in different subgroups was performed (Figure 1E). Patients in high-risk groups had a shorter OS time and higher death rate than those in low-risk groups. In addition, the area under the ROC curve (AUC) values of the prognostic signature for 1-, 3-, and 5 -year survival rates were 0.703, 0.689, and 0.652, respectively (Figure 1F). The AUC analysis revealed that prognostic signature was a substitute for other clinical factors in predicting LUAD patients' prognosis (Figure 1G).

\section{The validation of RHOV and other four hub MDGs in TCGA-LUAD dataset}

Next, we investigated the associations between RHOV and clinicopathological features of LUAD patients from TCGA-LUAD dataset. Compared to normal tissues, the mRNA expression level of RHOV was significantly increased in LUAD tissues (Figure 2A). RHOV mRNA expression was also increased in those with advanced clinical stage (Figure 2B), lymph node metastasis (Figure 2C), and large tumor size 
(Figure 2D). However, due to the small sample size, the association between RHOV and distant metastasis could not be analyzed. The associations between other four hub MDGs and clinicopathological features were also examined. Compared to normal tissues, CYP4B1 mRNA level was significantly decreased (Figure S1A), and GPR18, TCP10L2 and ZIC5 mRNA level were significantly increased in LUAD tissues (Figure S1B-D). CYP4B1 mRNA level was decreased in those with advanced clinical stage (Figure S1E). There were limited clinical values of GPR18, TCP10L2, and ZIC5 mRNA level, because of non-consistent expression level or non-significance in different clinical stages (Figure S1F-H).
A
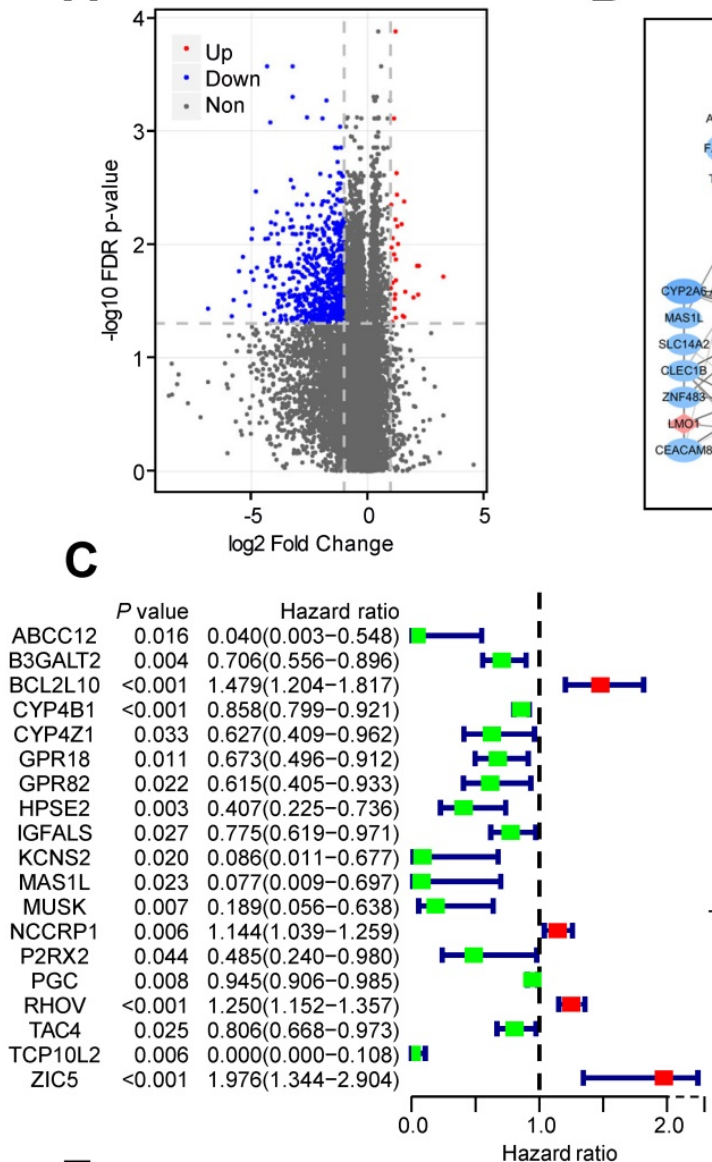

E
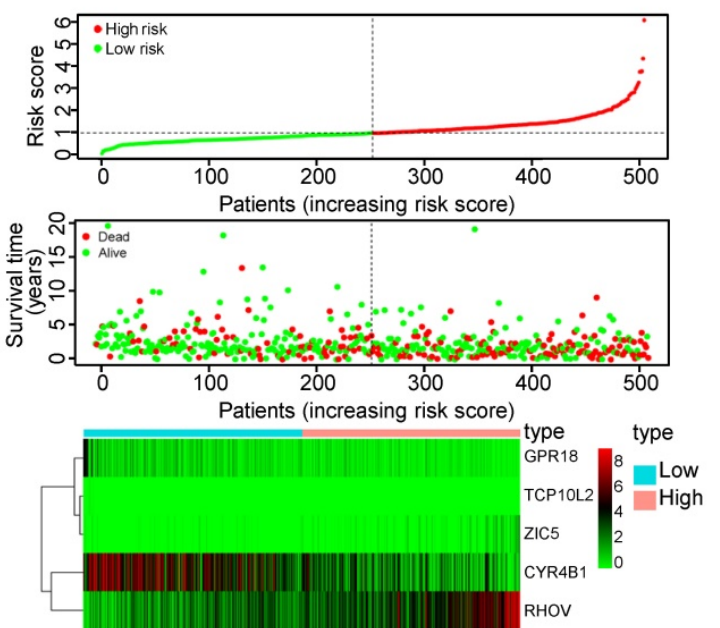

B

D
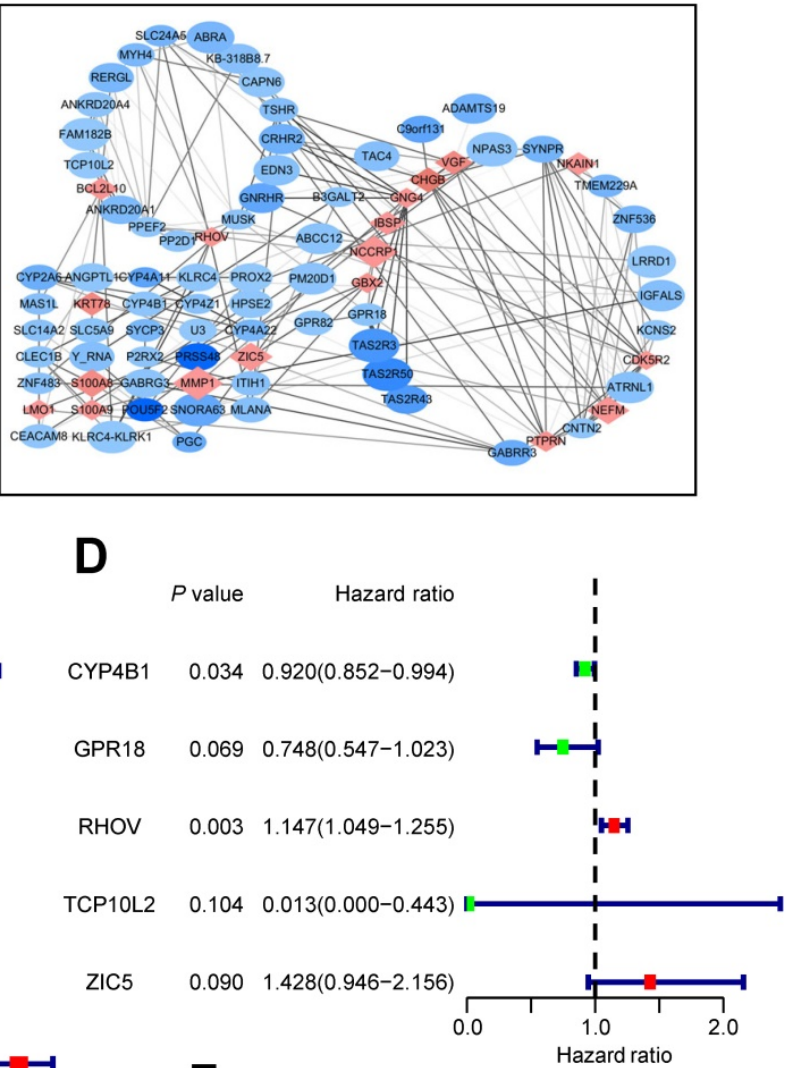

$\mathbf{F}$

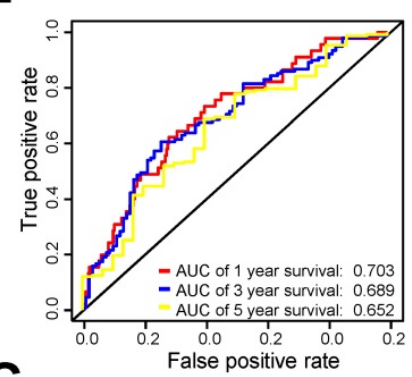

G

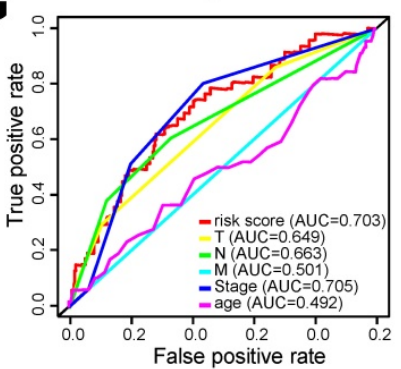

Figure 1. Identification of RHOV as the most significant gene associated with LUAD metastasis. (A) The volcano plot showing differentially expressed genes between tumor tissues with non-metastatic predisposition and tumor tissues with metastatic predisposition from TCGA-LUAD dataset. (B) A PPI network analysis of 83 MDGs. (C and D) The hazard ratio (HR) and $95 \%$ confidence interval of genes were calculated by univariate (C) and multivariate (D) Cox regression analysis. (E) The distribution of risk score, survival status and gene expression of LUAD patients from TCGA-LUAD dataset. (F and G) ROC analysis of the prognostic signature with 1-, 3- and 5-year overall survival and clinicopathological features with 1-year overall survival of LUAD patients. 

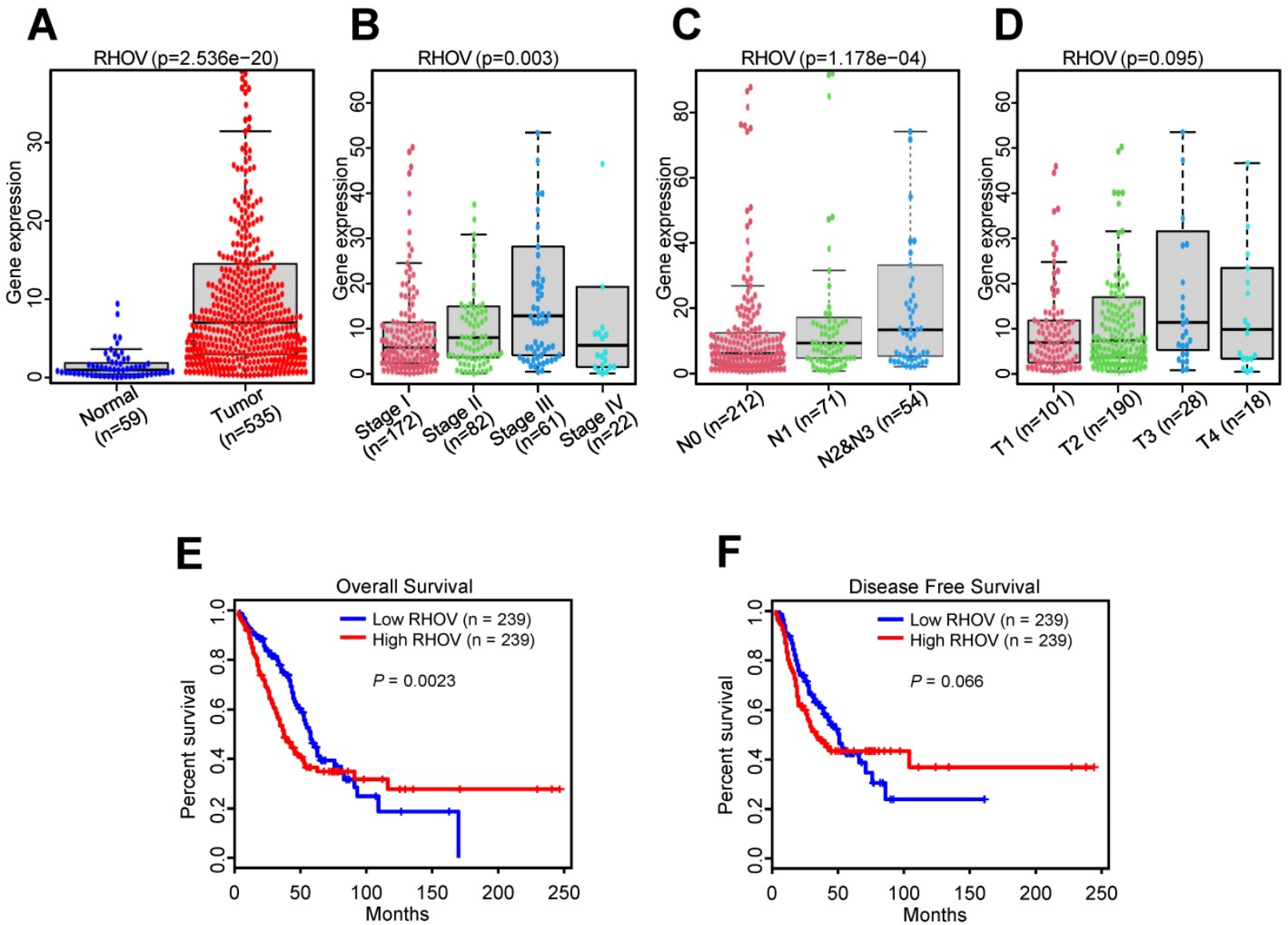

G
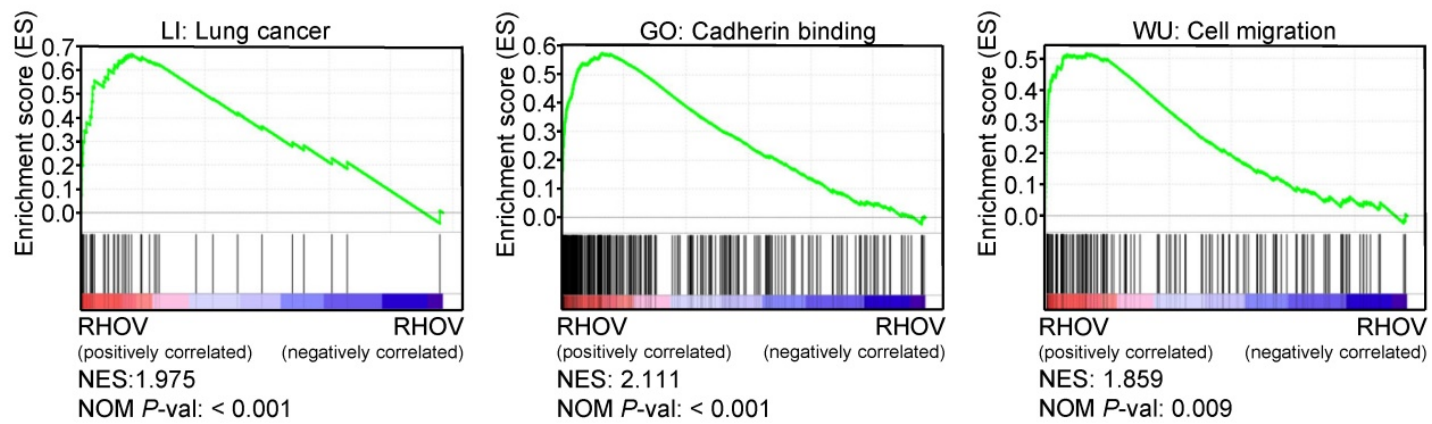

Figure 2. The validation and function prediction of RHOV in LUAD patients from TCGA-LUAD dataset. (A-D) The associations between RHOV mRNA expression and clinicopathological features in LUAD patients from TCGA-LUAD dataset. (E and F) The associations between RHOV mRNA expression and overall survival (E) and disease-free survival (F) in LUAD patients from TCGA-LUAD dataset. (G) GSEA was conducted to predict the function of RHOV in LUAD.

In the first 50 months of LUAD, high RHOV mRNA expression was associated with shorter OS in LUAD patients (Figure 2E). After 50 months, the negative correlation between RHOV mRNA expression and OS disappeared in LUAD patients. These results suggest that RHOV may play a key role as an oncogene in the early LUAD. As for the relation between RHOV and DFS in LUAD, similar phenomenon was found (Figure 2F). The associations between other four hub MDGs and prognosis of LUAD patients were also analyzed. CYP4B1 mRNA expression was positively correlated with $\mathrm{OS}$ in LUAD patients, and GPR18 and ZIC5 mRNA expression were negatively correlated with OS (Figure S2A-C). There was no significant correlation between mRNA expression of these MDGs and DFS in LUAD patients (Figure S2D-F).

To explore the function of RHOV in LUAD, gene set enrichment analysis (GSEA) was performed. The result showed that several pathways associated with LUAD metastasis, such as cadherin binding and cell migration, were significantly enriched (Figure 2G). 
A

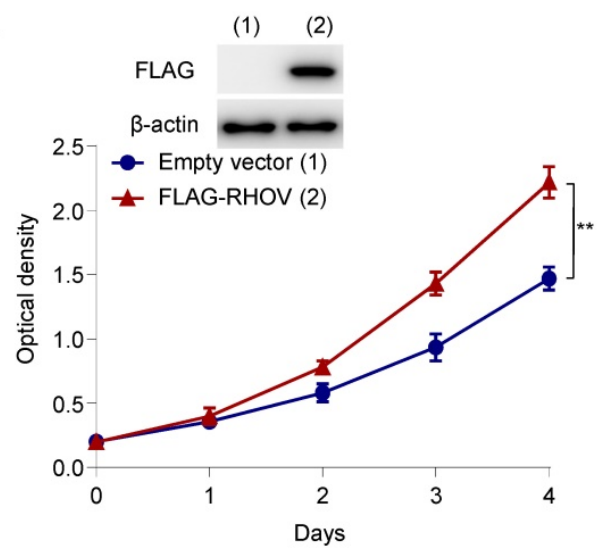

C

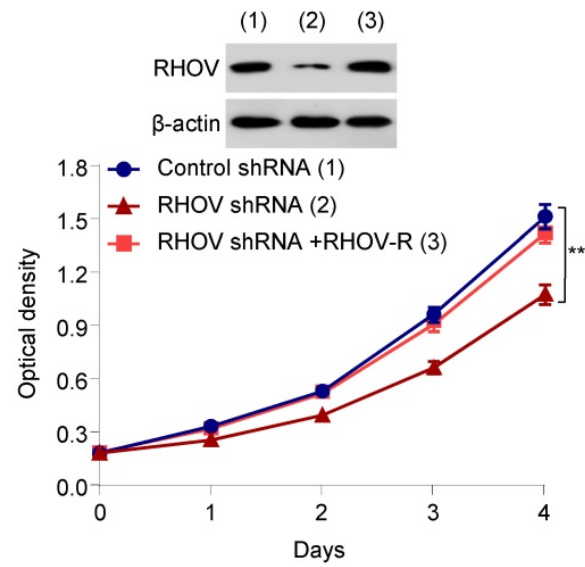

B

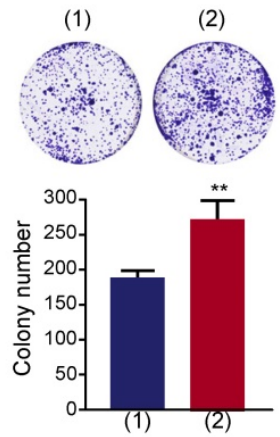

D
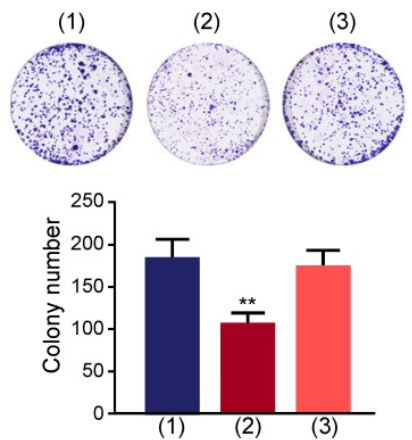

Figure 3. RHOV promotes cell proliferation in A549 cells. (A) A549 cells infected with FLAG-tagged RHOV plasmid or empty vector were grown in regular medium. Cell numbers were detected at the indicated times. Representative immunoblot demonstrates the expression of FLAG-RHOV. (B) Colony formation assays for A549 cells infected as in (A). (C) A549 cells were infected with control shRNA, RHOV shRNA or RHOV shRNA plus siRNA-resistant RHOV (RHOV-R), and analyzed as in (A). Representative immunoblot shows RHOV expression. (D) Colony formation assays for A549 cells infected as in (C). Data shown are mean \pm SD of triplicate measurements with similar results $(* P<0.05, * * P<0.01)$.

\section{RHOV promotes LUAD cell proliferation, migration and invasion in vitro}

Next, we investigated the function of RHOV on proliferation, migration and invasive capacity of LUAD cells. We chose two LUAD cell lines (A549 and H1299 cells) to test the function of RHOV. The expression level of RHOV in H1299 cells was only a little higher than that in A549 cells (Figure S3A). Thus, we used the same cell line to overexpression or knockdown of RHOV. Cell proliferation and colony formation assays revealed that FLAG-tagged RHOV-overexpressing A549 cells grew faster than empty vector-containing cells (Figure $3 \mathrm{~A}$ and $3 \mathrm{~B}$ ). In contrast, RHOV knockdown reduced proliferation and colony formation of A549 cells, and reexpression of RHOV in the RHOV knockdown cells rescued this effect (Figure 3C and 3D). Similar effects were observed in H1299 cells infected with FLAG-RHOV or RHOV shRNA plasmids (Figure S3B-E).

Wound-healing showed that RHOV overexpression promotes A549 cell migration (Figure 4A). Transwell assays demonstrated that RHOV overexpression increased the number of invaded A549 cells (Figure 4B). In contrast, RHOV knockdown reduced A549 cell migration and invasion, and reexpression of RHOV in the RHOV knockdown cells rescued this effect (Figure 4C and 4D). Similar effects were observed in H1299 cells (Figure S4). These data demonstrated that RHOV promotes LUAD cell proliferation, migration and invasion in vitro.

\section{JNK pathway is responsible for RHOV regulating LUAD cell proliferation, migration, invasion as well as the expression of EMT-related genes}

It has been reported that RHOV activate JNK pathway in human embryonic kidney 293 cells and rat pheochromocytoma PC12 cells [14, 15]. However, whether RHOV stimulates JNK pathway in human cancer cells is unknown. JNK pathway was known to be an essential role in lung cancer cell survival and metastasis $[16,17]$. Therefore, to study the mechanism by which RHOV regulates LUAD cell proliferation, migration, invasion, we explored the relationship between RHOV and JNK pathway in LUAD cells. 
RHOV overexpression activated phosphorylation of JNK and its downstream target c-Jun, without the changes of expression levels of total JNK and c-Jun in A549 cells (Figure 5A). Pyrazolanthrone, a JNK inhibitor, abrogated RHOV-promoted phosphorylation of JNK and c-Jun. Since EMT is critical for invasion and metastasis of cancer cells [18], we tested the effects of RHOV on EMT in LUAD cells. RHOV overexpression decreased expression of the epithelial marker E-cadherin, and increased that of the mesenchymal marker $\mathrm{N}$-cadherin and the EMT-related transcriptional factors Snail and Slug. However, RHOV overexpression did not change expression of Vimentin, another EMT-related protein, suggesting that RHOV specifically regulates the expression of E-cadherin and N-cadherin. Intriguingly, pyrazolanthrone treatment could also abrogate RHOV-dependent changes of EMT-related proteins (Figure 5A). Cell proliferation and colony formation assays showed that pyrazolanthrone abrogated RHOV promotion of cell proliferation of A549 cells (Figure 5B and 5C). Wound-healing and transwell assays showed that pyrazolanthrone
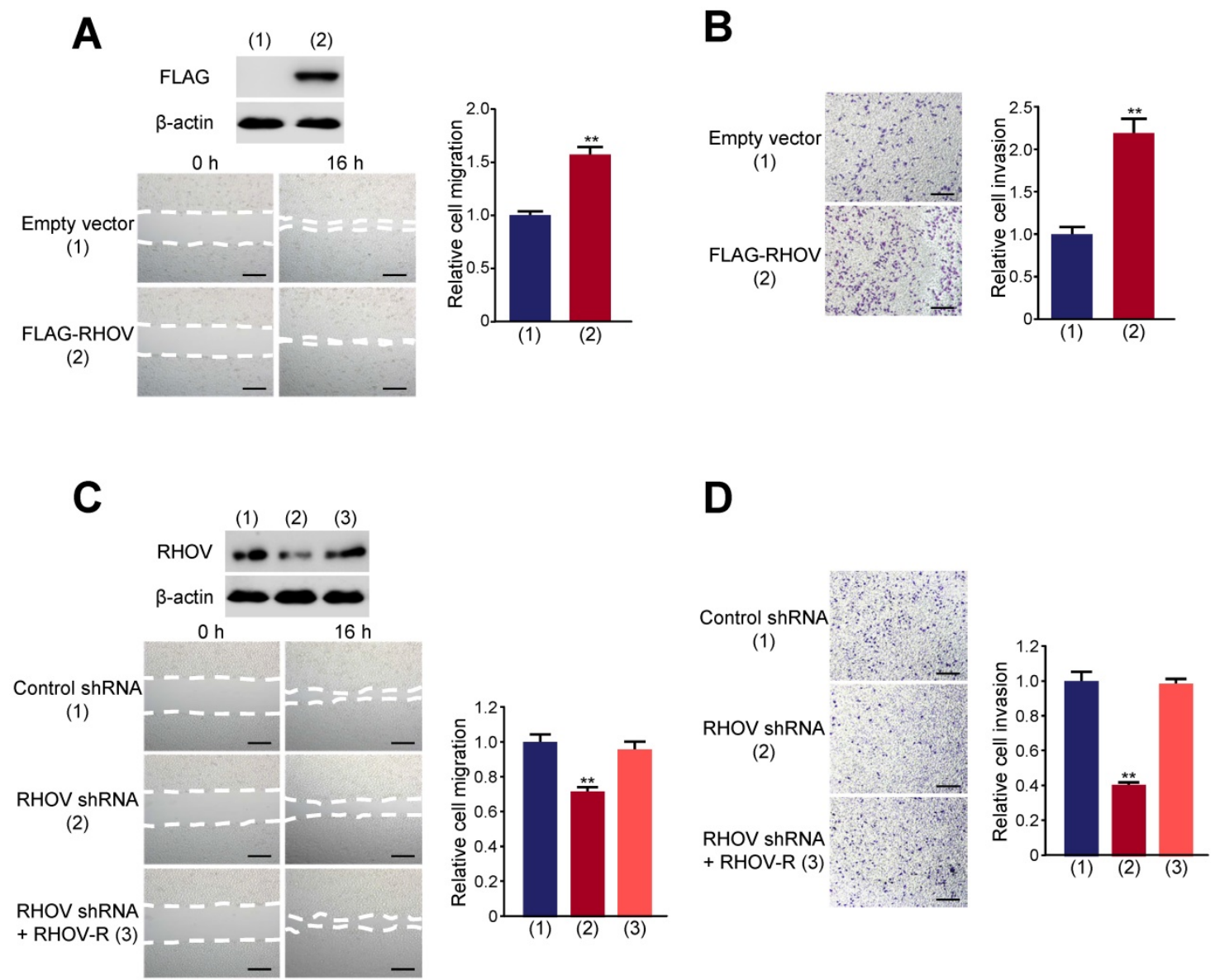

Figure 4. RHOV promotes migration and invasion in A549 cells. (A and B) Migration (A) and invasion (B) of A549 cells overexpressing FLAG-tagged RHOV plasmid or empty vector were evaluated by wound-healing assays and transwell assays, respectively. Scale bar: $100 \mu$ m. Representative immunoblot demonstrates the expression of FLAG-RHOV. (C and D) Migration (C) and invasion (D) of A549 cells infected with control shRNA, RHOV shRNA or RHOV shRNA plus siRNA-resistant RHOV (RHOV-R) were evaluated by wound-healing assays and transwell assays, respectively. Scale bar: $100 \mu \mathrm{m}$. Representative immunoblot shows RHOV expression. Data shown are mean \pm SD of triplicate measurements with similar results $(* P<0.05, * * p<0.01)$.

abrogated RHOV promotion of migration and invasion of A549 cells (Figure 5D and 5E). Similar results were also obtained in H1299 cells (Figure S5). Taken together, these results suggested that RHOV promotes LUAD cell proliferation, migration, invasion, and regulates the expression of EMT-related genes, via JNK pathway.

\section{Knockdown of RHOV suppresses LUAD tumor}

As RHOV promotes LUAD cell proliferation in vitro, we investigated whether RHOV modulates LUAD tumor growth in nude mice. A549 cells stably expressing RHOV shRNA or control shRNA were injected subcutaneously in the right flank of each nude mice. Compared with control shRNA, the tumors with RHOV knockdown grew slowly (Figure 6A). As expected, the A549 tumors in mice inoculated with RHOV shRNA decreased phosphorylation of JNK and c-Jun and expression of N-cadherin, Snail and Slug, and increased expression of E-cadherin (Figure 6B).

D 
A

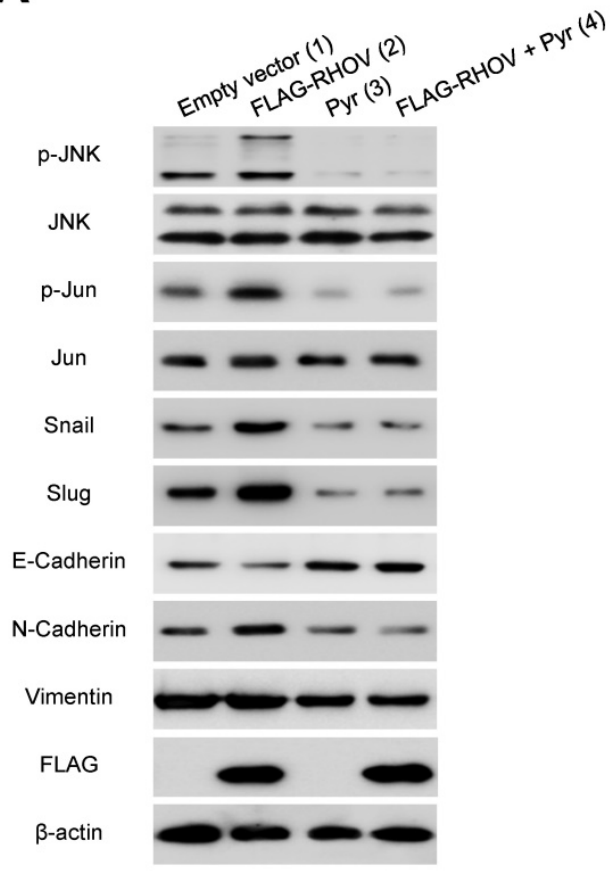

D

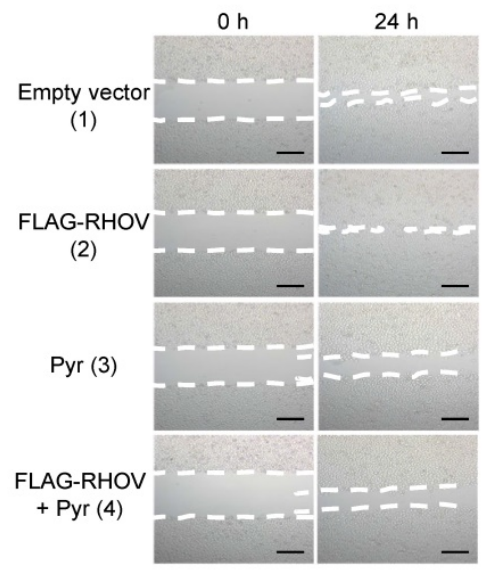

B

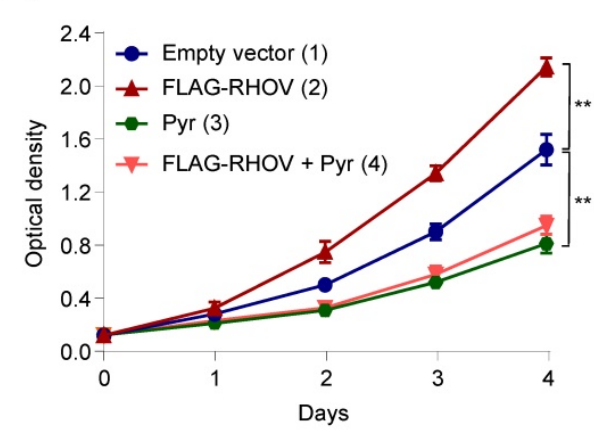

C

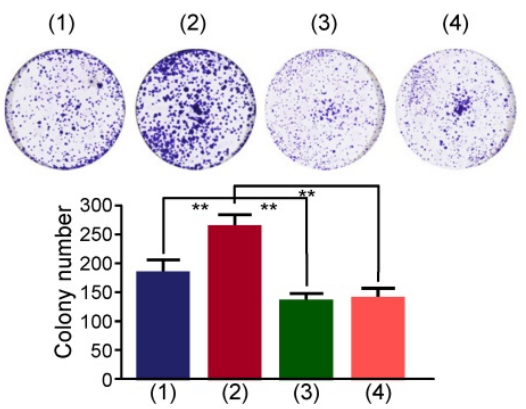

E

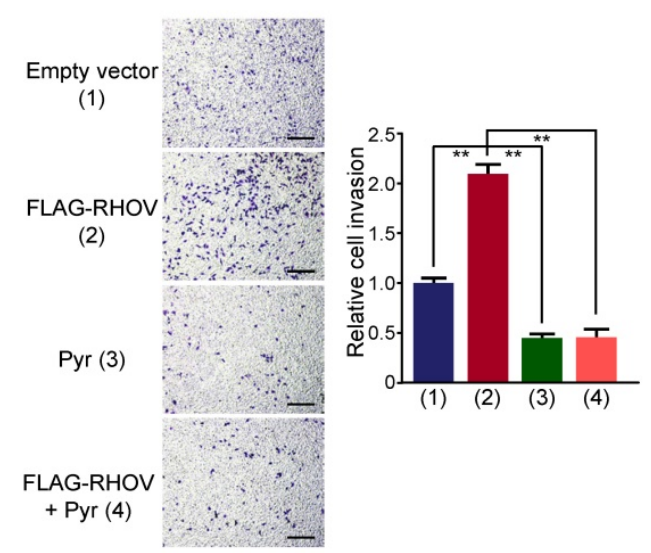

Figure 5. RHOV regulates expression of EMT-related proteins via JNK pathway. (A) Western blot analysis of A549 cells infected with FLAG-tagged RHOV plasmid and treated with the JNK inhibitor pyrazolanthrone $(10 \mu \mathrm{M})$. (B and C) A549 cells were treated as in (A), and cell numbers (B) and cell colonies (C) were detected at the indicated times. (D and E) Migration (D) and invasion (E) of A549 cells treated as in (A) were evaluated by wound-healing and transwell assays, respectively. Scale bar: $100 \mu \mathrm{m}$. Data shown are mean \pm SD of triplicate measurements with similar results $(* P<0.05, * * P<0.01)$.

Since RHOV promotes LUAD cell migration and invasion in vitro, we explored whether RHOV regulates LUAD metastasis. The control and RHOV knockdown A549 cells were suspended in PBS and injected into nude mice via the tail vein. Eight weeks after the injection, H\&E staining images of lung metastases demonstrated that RHOV knockdown significantly decreased metastasis of A549 cells (Figure 6C). Taken together, these data suggests that RHOV knockdown inhibits tumor growth and metastasis of LUAD in vivo.

\section{Discussion}

In this study, five hub genes, including RHOV,
ZIC5, CYP4B1, GPR18 and TCP10L2, have been identified as LUAD metastasis driver genes. Univariate and multivariate Cox regression analysis suggest that RHOV is the most significant gene associated with prognosis of LUAD patients among five genes. RHOV is overexpressed in LUAD patients, and high RHOV expression correlates with large tumor size, advanced clinical stage, lymph node metastasis, and shorter OS. Furthermore, we show that RHOV promotes LUAD cell proliferation, migration, invasion and metastasis both in vitro and in vivo. The JNK inhibitor pyrazolanthrone diminishes the effect of RHOV on LUAD cell proliferation, migration and invasion, suggesting that RHOV 
A
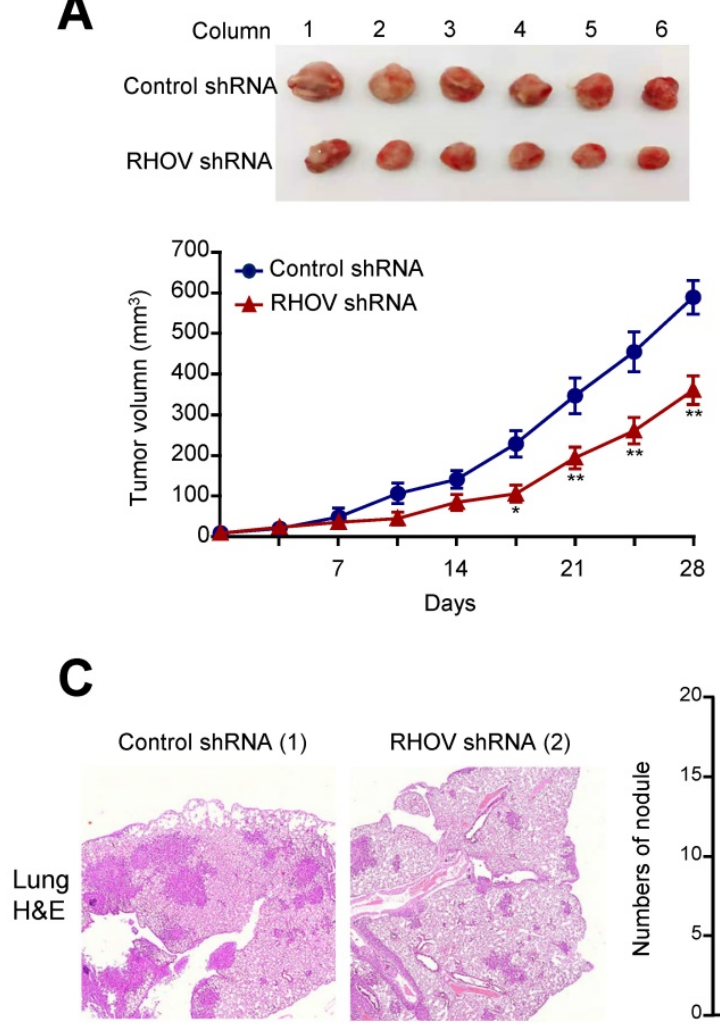

D
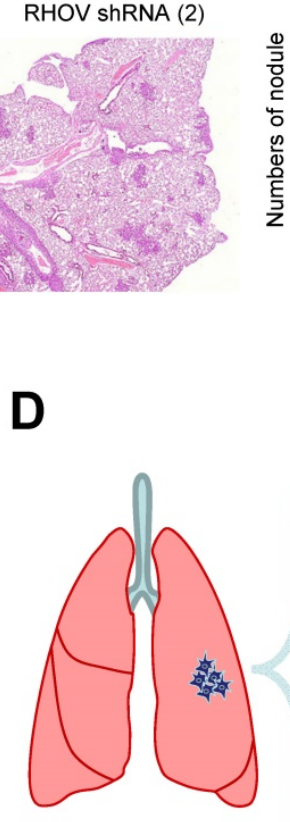

B

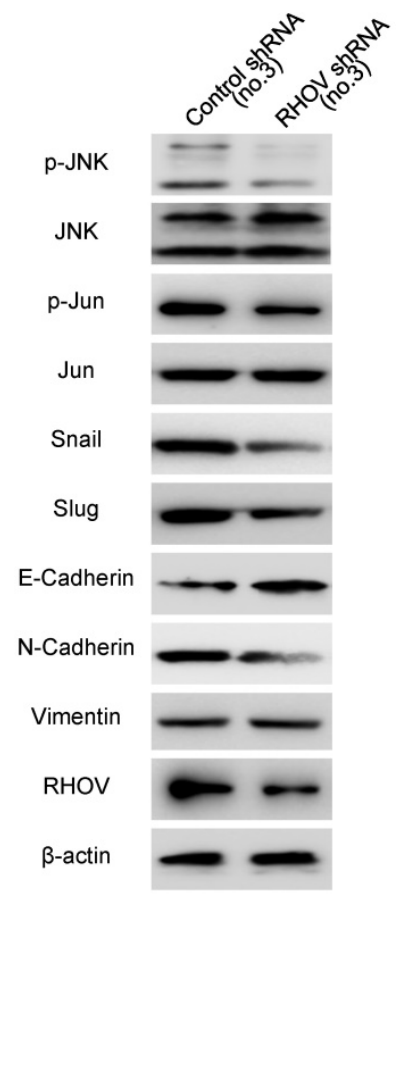

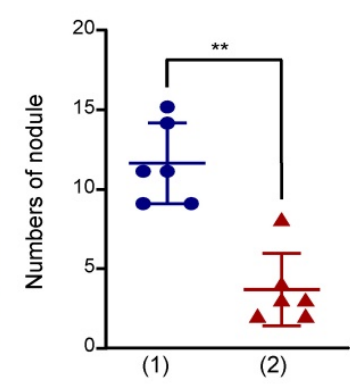

RHOV

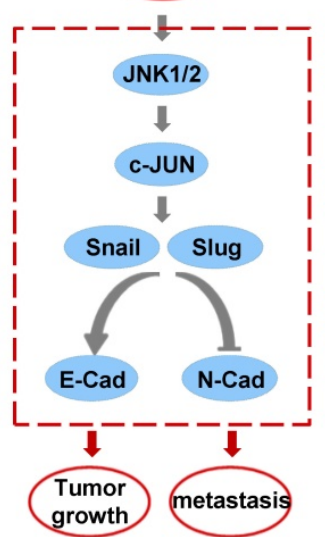

Figure 6. Knockdown of RHOV suppresses LUAD tumor growth and metastasis in nude mice. (A) A549 cells stably infected with RHOV shRNA or control shRNA were injected subcutaneously in the right flank of nude mice. At the indicated times, the volume of the tumors was examined with Vernier calipers. (B) Immunoblot analysis of representative excised tumor tissues from (A). (C) Representative H\&E-stained sections of the lung tissues are shown. Data are shown as mean $\pm S D(n=6)(* P<0.05, * * P<0.01$ versus control shRNA).

regulates LUAD cell proliferation, migration and invasion via JNK pathway. Thus, our study identifies RHOV as a critical gene responsible for LUAD growth and metastasis, and RHOV may be an ideal target for LUAD therapy.

RHOV is a member of the Rho GTPase family, and plays a vital role in neurodevelopment and embryogenesis $[19,20]$. RHOV have been identified to activate p21-activated kinases (Paks) [9, 21], which affect a wide range of physiological and pathological processes [22]. However, the biological function of
RHOV in human cancer is unclear. We show that RHOV controls human LUAD growth and metastasis. JNK is typical serine/threonine kinases and regulates diverse aspects of tumorigenesis and progression, including proliferation, survival and metastasis [23-25]. JNK pathway maintains various capabilities of cancer stem cell properties, including self-renewal, drug resistance, and tumorigenesis [26]. JNK promotes tumor metastasis via disintegrating adherent junctions [27]. The transcription factor c-Jun phosphorylation was identified to be an essential 
prerequisite for the activation of JNK pathway [17]. Phosphorylation of c-Jun can enhance its binding to the promoters of oncogenes, thereby increasing its transcriptional activity [28]. EMT, a process involved in tumor metastasis, can also be induced by JNK pathway [29]. In this study, we demonstrated that RHOV acts as an oncogene by increasing expression of the EMT-related proteins N-cadherin, Snail and Slug, and decreasing that of the EMT-related protein E-cadherin. Importantly, we revealed for the first time that RHOV promotes growth, EMT and metastasis through JNK phosphorylation in human cancer. Whether RHOV regulates cancer cell growth and metastasis through other signaling pathways remains to be investigated.

Besides RHOV, other four hub genes (ZIC5, CYP4B1, GPR18 and TCP10L2) were screened to be associated with LUAD metastasis in this study. ZIC5 was reported to be highly upregulated in non-small cell lung cancer (NSCLC) tissues and act as an oncogene through regulating the expression of cell cycle-related cdk1/cyclin B1 complex [30]. CYP4B1 is a xenobiotics metabolism enzyme, and participates in various biological processes, especially tumorigenesis $[31,32]$. Compared to normal tissues, CYP4B1 expression level is reduced in the lung tumors [33]. GPR18, a cannabinoid-stimulated G protein-coupled receptor, has been implicated in a variety of cancers, involving melanoma [34], breast cancer [35], oral cancer [36], and hepatocellular carcinoma [37]. However, the functions of GPR18 have not been yet investigated in lung cancer. TCP10L2 is considered as a pseudogene, and its function remains to be studied.

\section{Supplementary Material}

Supplementary figures and table.

http://www.ijbs.com/v17p2622s1.pdf

\section{Acknowledgements}

This work was supported by National Natural Science Foundation (81630067, 81930078 and 81872246).

\section{Competing Interests}

The authors have declared that no competing interest exists.

\section{References}

1. Tang $Q$, Li W, Zheng $X$, et al. MELK is an oncogenic kinase essential for metastasis, mitotic progression, and programmed death in lung carcinoma. Signal Transduct Target Ther. 2020; 5(1): 279

2. Zhang H, Guo L, and Chen J. Rationale for Lung Adenocarcinoma Prevention and Drug Development Based on Molecular Biology During Carcinogenesis. Onco Targets Ther. 2020; 13: 3085-3091.

3. Relli V, Trerotola M, Guerra E, et al. Abandoning the Notion of Non-Small Cell Lung Cancer. Trends Mol Med. 2019; 25(7): 585-594.

4. Anichini A, Perotti VE, Sgambelluri F, et al. Immune Escape Mechanisms in Non Small Cell Lung Cancer. Cancers (Basel). 2020; 12(12).
5. Sholl LM, Aisner DL, Varella-Garcia M, et al. Multi-institutional Oncogenic Driver Mutation Analysis in Lung Adenocarcinoma: The Lung Cancer Mutation Consortium Experience. J Thorac Oncol. 2015; 10(5): 768-777.

6. Gomperts BN, Walser TC, Spira A, et al. Enriching the molecular definition of the airway "field of cancerization:" establishing new paradigms for the patient at risk for lung cancer. Cancer Prev Res (Phila). 2013; 6(1): 4-7.

7. Hodge RG and Ridley AJ. Regulation and functions of RhoU and RhoV. Small GTPases. 2020; 11(1): 8-15.

8. Faure $\mathrm{S}$ and Fort P. Atypical RhoV and RhoU GTPases control development of the neural crest. Small GTPases. 2015; 6(4): 174-7.

9. Tay HG, Ng YW, and Manser E. A vertebrate-specific Chp-PAK-PIX pathway maintains E-cadherin at adherens junctions during zebrafish epiboly. PLoS One. 2010; 5(4): e10125.

10. Shepelev MV and Korobko IV. The RHOV gene is overexpressed in human non-small cell lung cancer. Cancer Genet. 2013; 206(11): 393-7.

11. Cerami E, Gao J, Dogrusoz U, et al. The cBio cancer genomics portal: an open platform for exploring multidimensional cancer genomics data. Cancer Discov. 2012; 2(5): 401-4.

12. Ritchie ME, Phipson $B, W u$ D, et al. limma powers differential expression analyses for RNA-sequencing and microarray studies. Nucleic Acids Res. 2015; 43(7): e47.

13. Shukla S, Evans JR, Malik R, et al. Development of a RNA-Seq Based Prognostic Signature in Lung Adenocarcinoma. J Natl Cancer Inst. 2017; 109(1).

14. Aronheim A, Broder YC, Cohen A, et al. Chp, a homologue of the GTPase $\mathrm{Cdc} 42 \mathrm{Hs}$, activates the JNK pathway and is implicated in reorganizing the actin cytoskeleton. Curr Biol. 1998; 8(20): 1125-8.

15. Shepelev MV, Chernoff J, and Korobko IV. Rho family GTPase Chp/RhoV induces PC12 apoptotic cell death via JNK activation. Small GTPases. 2011; 2(1): 17-26.

16. Kamiyama M, Shirai $\mathrm{T}$, Tamura $\mathrm{S}$, et al. ASK1 facilitates tumor metastasis through phosphorylation of an ADP receptor P2Y12 in platelets. Cell Death Differ. 2017; 24(12): 2066-2076.

17. Wu Q, Wu W, Fu B, et al. JNK signaling in cancer cell survival. Med Res Rev. 2019; 39(6): 2082-2104

18. Ramesh V, Brabletz T, and Ceppi P. Targeting EMT in Cancer with Repurposed Metabolic Inhibitors. Trends Cancer. 2020; 6(11): 942-950.

19. Notarnicola C, Le Guen L, Fort P, et al. Dynamic expression patterns of RhoV/Chp and RhoU/Wrch during chicken embryonic development. Dev Dyn. 2008; 237(4): 1165-71.

20. Guemar L, de Santa Barbara P, Vignal E, et al. The small GTPase RhoV is an essential regulator of neural crest induction in Xenopus. Dev Biol. 2007; 310(1): 113-28.

21. Shepelev MV and Korobko IV. Pak6 protein kinase is a novel effector of an atypical Rho family GTPase Chp/RhoV. Biochemistry (Mosc). 2012; 77(1): 26-32.

22. Yao D, Li C, Rajoka MSR, et al. P21-Activated Kinase 1: Emerging biological functions and potential therapeutic targets in Cancer. Theranostics. 2020; 10(21): 9741-9766.

23. Chen F. JNK-induced apoptosis, compensatory growth, and cancer stem cells. Cancer Res. 2012; 72(2): 379-86.

24. Peluso I, Yarla NS, Ambra R, et al. MAPK signalling pathway in cancers: Olive products as cancer preventive and therapeutic agents. Semin Cancer Biol. 2019; 56: 185-195.

25. Weston CR and Davis RJ. The JNK signal transduction pathway. Curr Opin Cell Biol. 2007; 19(2): 142-9.

26. Semba T, Sammons R, Wang X, et al. JNK Signaling in Stem Cell Self-Renewal and Differentiation. Int J Mol Sci. 2020; 21(7)

27. Lee MH, Padmashali $\mathrm{R}$, Koria $\mathrm{P}$, et al. JNK regulates binding of alpha-catenin to adherens junctions and cell-cell adhesion. FASEB J. 2011; 25(2): 613-23.

28. Spigolon G, Cavaccini A, Trusel M, et al. cJun N-terminal kinase (JNK) mediates cortico-striatal signaling in a model of Parkinson's disease. Neurobiol Dis. 2018; 110: 37-46.

29. Pang B, Wu N, Guan R, et al. Overexpression of RCC2 Enhances Cell Motility and Promotes Tumor Metastasis in Lung Adenocarcinoma by Inducing Epithelial-Mesenchymal Transition. Clin Cancer Res. 2017; 23(18): 5598-5610.

30. Sun Q, Shi R, Wang X, et al. Overexpression of ZIC5 promotes proliferation in non-small cell lung cancer. Biochem Biophys Res Commun. 2016; 479(3): 502-509.

31. Jarrar YB and Lee SJ. Molecular Functionality of Cytochrome P450 4 (CYP4) Genetic Polymorphisms and Their Clinical Implications. Int J Mol Sci. 2019; 20(17).

32. Baer BR and Rettie AE. CYP4B1: an enigmatic P450 at the interface between xenobiotic and endobiotic metabolism. Drug Metab Rev. 2006; 38(3): 451-76.

33. Czerwinski M, McLemore TL, Gelboin HV, et al. Quantification of CYP2B7, CYP4B1, and CYPOR messenger RNAs in normal human lung and lung tumors. Cancer Res. 1994; 54(4): 1085-91.

34. Qin $Y$, Verdegaal EM, Siderius M, et al. Quantitative expression profiling of G-protein-coupled receptors (GPCRs) in metastatic melanoma: the constitutively active orphan GPCR GPR18 as novel drug target. Pigment Cell Melanoma Res. 2011; 24(1): 207-18.

35. Tomko A, O'Leary $\mathrm{L}$, Trask $\mathrm{H}$, et al. Antitumor Activity of Abnormal Cannabidiol and Its Analog O-1602 in Taxol-Resistant Preclinical Models of Breast Cancer. Front Pharmacol. 2019; 10: 1124 
36. Ye $\mathrm{Y}, \mathrm{Scheff} \mathrm{NN}$, Bernabe $\mathrm{D}$, et al. Anti-cancer and analgesic effects of resolvin D2 in oral squamous cell carcinoma. Neuropharmacology. 2018; 139: 182-193.

37. Qiao GJ, Chen $\mathrm{L}, \mathrm{Wu} J \mathrm{C}$, et al. Identification of an eight-gene signature for survival prediction for patients with hepatocellular carcinoma based on integrated bioinformatics analysis. PeerJ. 2019; 7: e6548. 